\title{
Analysis of the Functionality of the Transformer Ripple Filter for High- power DC Supplies
}

\author{
Lokvenc Jaroslav, Drtina Rene and Sedivy Josef \\ Department of Technical Subjects, Faculty of Education, University of Hradec Kralove, Rokitanskeho 62, 50003 Hradec Kralove, Czech \\ Repubilc
}

\begin{abstract}
In the field of DC power supplies stabilized classic analog circuits, which are mainly in the last twenty years has been increasingly squeezed by switching technology and digital control. Implementation of the research project stabilized linear power supply to heavy traffic conditions, which is the primary requirement for high operational reliability, low noise voltage at the output of the power supply and the high overload capacity, bring DC power supply transformer developed with us compensate for output ripple. Article presents analysis of the Functionality of the transformer ripple filter for DC power supplies.
\end{abstract}

\section{Introduction}

Each solution DC power supplies (analog/switching) has its advantages and disadvantages [1]. Standard analog sources usually tolerate temporary overload of $10-15 \%$ over several minutes. In industrial applications, it is then necessary for the design resources into account not only the nature of the load and possible overloading (short term and over $100 \%$ ), but also the operating conditions in the environment (dust, humidity, temperature fluctuations, condensation, aggressive environment). The aim of the research project was the realization of a functional sample stabilized linear power supply to heavy operating conditions, the output current-voltage characteristic would be comparable to current-voltage characteristic of leadacid battery.

Our proposed DC power supplies are designed for applications that do not mind a slight variation of the output voltage with the load, but the primary requirement is high operational reliability, low noise voltage at the output of the power supply and a substantial (and long term) capability. For filtering the output voltage using DC power supplies developed by us compensation transformer output ripple. DC power supplies are designed for heavy-duty industrial applications, are composed almost exclusively of passive elements and use conventional cooling.

\section{Design of transformer ripple filter}

Motion power filter compensation transformer are first published in connection with the construction of the single-phase linear unconventional sources [2] (Fig. 1) and then modified for three-phase linear high-power DC supply [3] (Fig. 2). The proposed solution does not use conventional technology or lower bandpass filters, but subtraction disruptive ripple from power.

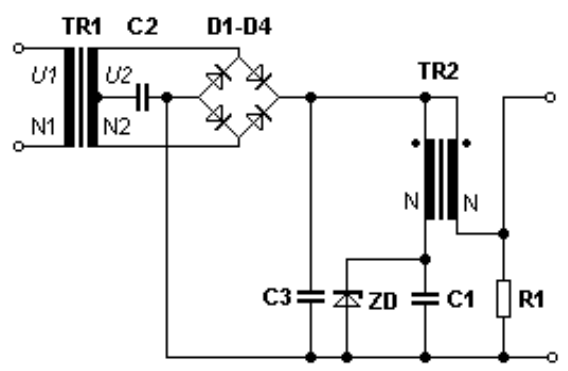

Figure 1. One-phase DC supply (by [1]).

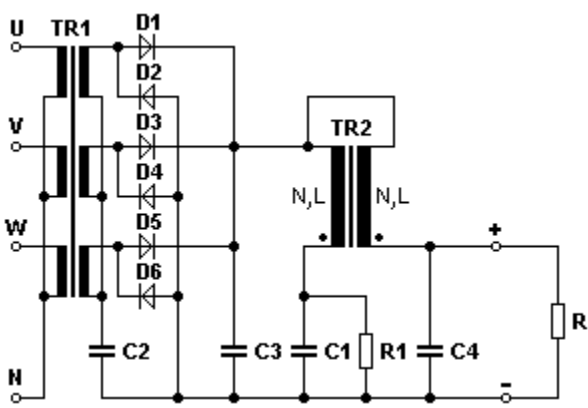

Figure 2. Tree-phase DC supply (by [2]).

The voltage at the output single-phase rectifier is by Fourier-shaped

$$
\begin{aligned}
& U(\omega t)=\frac{4 U_{\mathrm{m}}}{\pi} \cdot\left(\frac{1}{2}-\frac{1}{3} \cos 2 \omega t-\frac{1}{3 \cdot 5} \cos 4 \omega t-\right. \\
& \left.-\frac{1}{5 \cdot 7} \cos 6 \omega t-\frac{1}{7 \cdot 9} \cos 8 \omega t \ldots\right)
\end{aligned}
$$

and the amplitude of the second harmonic $U_{2 \mathrm{~h}}$ 


$$
U_{2 \mathrm{hm}}=\frac{4 U_{\mathrm{m}}}{\pi} \cdot \frac{1}{3}
$$

The voltage at the output of a three-phase rectifier according to Fourier shaped

$$
\begin{aligned}
& U(\omega t)=\frac{3 U_{\mathrm{m}} \sqrt{3}}{\pi} \cdot\left(1-\frac{2}{5 \cdot 7} \cos 6 \omega t-\right. \\
& \left.\frac{2}{11 \cdot 13} \cos 12 \omega t-\frac{2}{17 \cdot 19} \cos 18 \omega t-\ldots\right)
\end{aligned}
$$

and the amplitude of the sixth harmonic (with respect to power frequency) $U_{6 \mathrm{hm}}$

$$
U_{6 \mathrm{hm}}=\frac{3 U_{\mathrm{m}} \sqrt{3}}{\pi} \cdot \frac{2}{5 \cdot 7}
$$

Compensation transformer is compared to the current practice designed for a closed magnetic core, which is a DC power supply output current source. Output current source $I_{\mathrm{DC}}$ calls the core of the transformer TR2 compensating coil with inductance $L_{\mathrm{DC}}$ induction sizes

$$
B_{\mathrm{DC}}=\frac{\mu_{0} \mu_{\mathrm{r}} N I_{\mathrm{DC}}}{l_{\mathrm{s}}}
$$

and magnetizing current $I_{\mu}$

$$
I_{\mu}=\frac{U_{2 \mathrm{hm}}}{4 \pi f L} \text { (for single-phase supply) }
$$

or

$$
I_{\mu}=\frac{U_{6 \mathrm{hm}}}{12 \pi f L}(\text { for three-phase source })
$$

Aggregate peak magnetic flux density in the core must be at maximum current satisfy according the condition

$$
B_{\max } \geq B_{\mathrm{DC}}+B_{\mu} \cdot \sqrt{2}
$$

\section{Transformer filter - analysis}

Transformer operated on alternating current transmission or distribution network transfers power from the primary winding $R_{1} X_{1 \sigma} X_{\mathrm{h}}$ (from terminals 1-1') to the magnetic circuit $R_{\mathrm{Fe}} X_{\mathrm{h}}$ into secondary winding $R_{2} X_{2 \sigma} X_{\mathrm{h}}$ (terminals $2-2^{\prime}$ ), wherein $R_{1}, R_{2}$ are resistors winding and $X_{1 \sigma}, X_{2 \sigma}$ leakage inductance. For solution we use a simplified diagram of a replacement, the T-cell with $p=1$ transfers without parasitic capacities (Fig. 3) [4]. Values $R_{2}, X_{2 \sigma}$ not necessary to recalculate in the scheme [5].

Magnetic induction in the iron core of the transformer is in the range $\pm B$, depending on the hysteresis loop of the material (Fig. 4), if it possible, in the linear part.

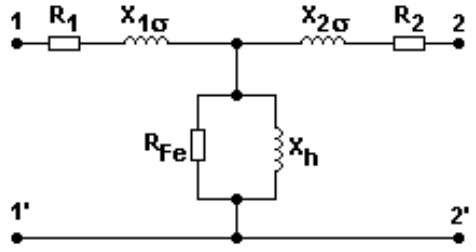

Figure 3. Equivalent diagram of transformer.

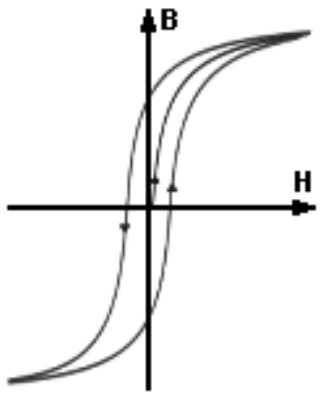

Figure 4. Hysteresis loop of transformer core.

By contrast, compensatory transfers from the transformer primary circuit (terminals 1-1' connect to the output rectifier) DC power to the secondary circuit (terminals 22 ') only through the secondary winding $R_{2} X_{2 \sigma} X_{2 \mathrm{~h}}$ (Fig. 5). The current through the secondary coil $X_{2 \mathrm{~h}}$ excite the core of transformer DC magnetic induction $B_{\mathrm{DC}}$ (Fig. 6) according to Eq. 5 .

Primary winding $R_{1} X_{1 \sigma} X_{1 \mathrm{~h}}$ is isolated DC from the out put of the rectifier capacitor $\mathrm{C}$. Windings flows through only the alternating component is superimposed to the primary coil $X_{1 \mathrm{~h}}$, excites the core of the compensation transformer magnetic flux density $\Delta B=2 \cdot B_{\mu}$ (Fig. 6) according to Eq. 5. The resulting induction in the core then varies between $B_{\mathrm{DC}} \pm B_{\mu} \cdot \sqrt{ } 2$. Considered optimal to the working area $\Delta B$ was relatively linear part of the magnetization curve.

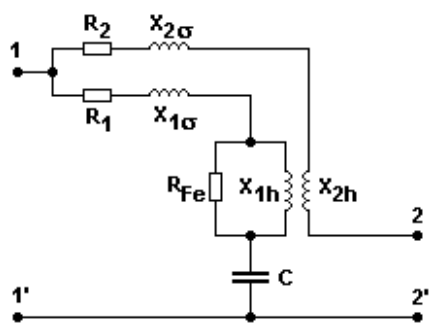

Figure 5. Equivalent diagram of compensation transformer.

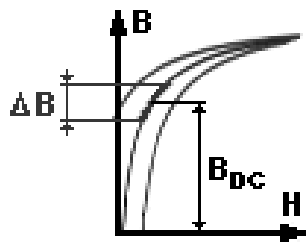

Figure 6. Magnetic induction in the core of compensation transformer.

Because the transformer ratio $p=1$ and the windings are formed as bifilar applies $R_{1}=R_{2}, X_{1 \sigma}=X_{2 \sigma}, X_{1 \mathrm{~h}}=X_{2 \mathrm{~h}}$. $R_{\mathrm{Fe}}$ are iron losses. However, they only creates alternating current component passing through the primary winding. 


\subsection{The influence of phase relationships of transformer operation filter}

In order compensation transformer totally eliminates disruptive ripple on the output power, it would have to meet three basic requirements:

1) $\mathrm{AC}$ voltage at the primary and secondary side with respect to section 1 (Fig. 5) the same size;

2) the alternating voltage on the primary and secondary side are of identical shape (transformer clogged shape distortion);

3) the $\mathrm{AC}$ voltage at the primary and secondary side with respect to each zero phase shift.

Conditions 1) and 2) can be met relatively without much difficulty. The voltage adjust slight change the number of turns of the secondary winding, shape distortion on the secondary side DC restrict core saturation and a low level alternating magnetization, preferably within a maximum linear extent permitted magnetic induction $B_{\max }$.

Issues voltage phase shift between the primary and secondary sides comes from the phasor diagrams loaded transformer. Zero phase shift in real magnetic circuits but virtually unattainable. Our effort is to reach a situation where $\varphi \rightarrow 0$. In practice, this means minimizing losses in the magnetic circuit. In the case of the compensation transformer, which transmits only the minimum power, it is possible to approximate this requirement. The results of simulating the impact of the phase shift between the primary and secondary voltage of the transformer compensation for the size differential voltage, which represents the spurious output are shown in Fig. 7.

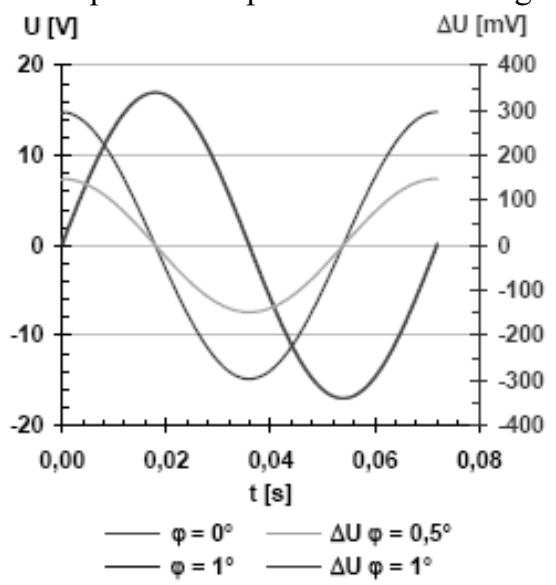

Figure 7. Simulation differential voltage.

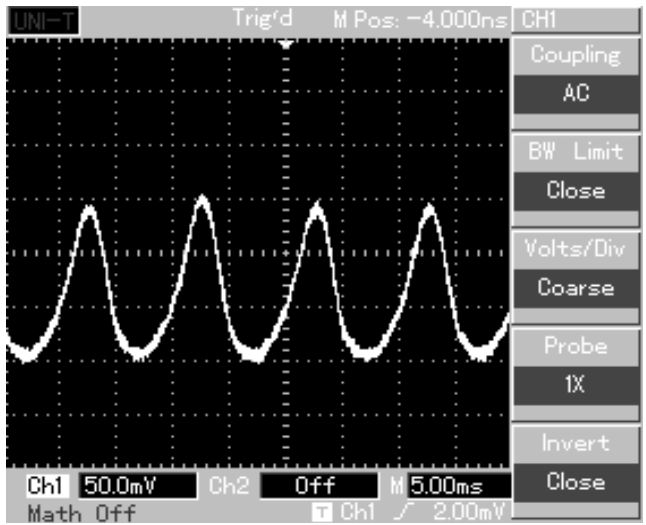

Figure 8. Oscillogram of the differential voltage.
For the amplitude of the input voltage $17 \mathrm{~V}$ singlephase source and the phase angle $\varphi=0,5^{\circ}$ based on the residual noise voltage with amplitude of $150 \mathrm{mV}$. When the phase shift $\varphi=1^{\circ}$, then the amplitude of the disturbing voltage of $300 \mathrm{mV}$. On compensation transformer developmental sample power source we are for direct consumption $I_{\mathrm{DC}}=10 \mathrm{~A}$ and measured peak to peak differential voltage $152 \mathrm{mV}$ (Fig. 8), which corresponds to the effective value of the ripple $48,5 \mathrm{mV}$ noise voltage at the output has a level of $-50 \mathrm{~dB}$.

\subsection{Results of transformer filter applications in power supplies}

Transformer ripple filter for high-power DC supplies have been long tested in laboratory and industrial use. Tests were run on linear single phase unstabilized supply $14,2 \mathrm{~V} / 10 \mathrm{~A}$ and unstabilized linear three-phase source of $13,2 \mathrm{~V} / 150 \mathrm{~A}$. Output current-voltage characteristic (Fig. 9, Fig. 10) shows good stability of the output voltage and a very small noise voltage at the output source.

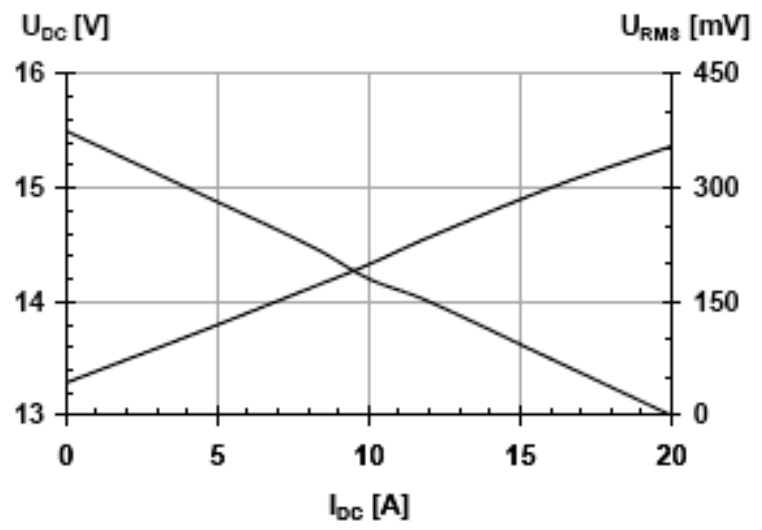

Figure 9. Output and ripple voltage (single-phase source)

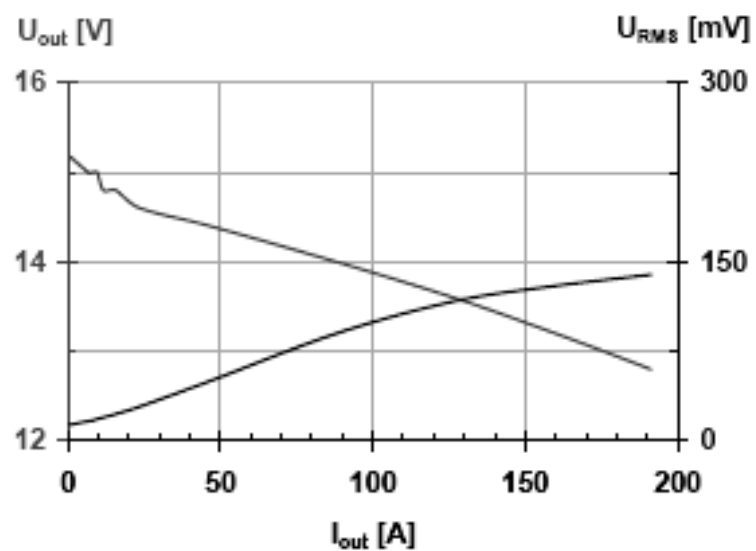

Figure 10. Output and ripple voltage (three-phase source).

Analysis of voltage drops on the individual parts of the source has proved a major impact on used rectifier output voltage drop. Used rectifier causes, depending on the load output voltage drop to $2 \mathrm{~V}$. Fig. 11 shows overlapping voltage transformer for compensation at a current of 200 A, on Fig. 12 shows the ripple at the input of the compensation transformer $(\mathrm{CH} 1)$ and the output ripple DC voltage ( $\mathrm{CH} 2)$. 


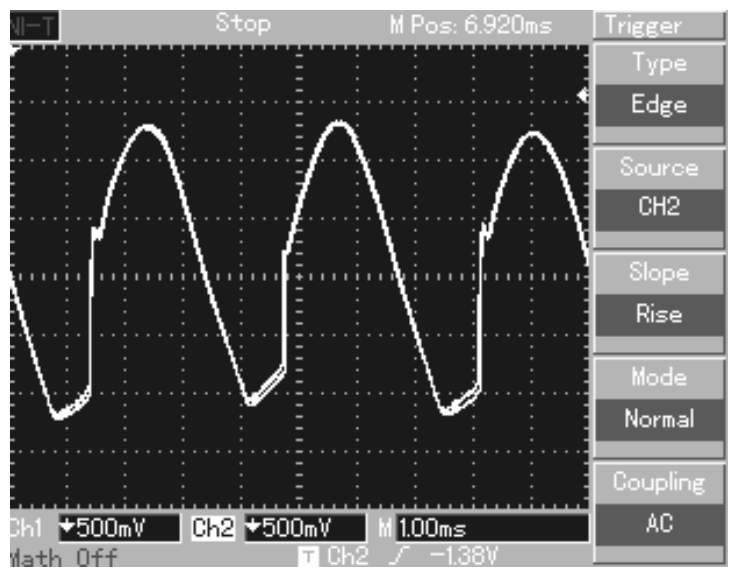

Figure 11. Overlapping voltage on transformer filter.

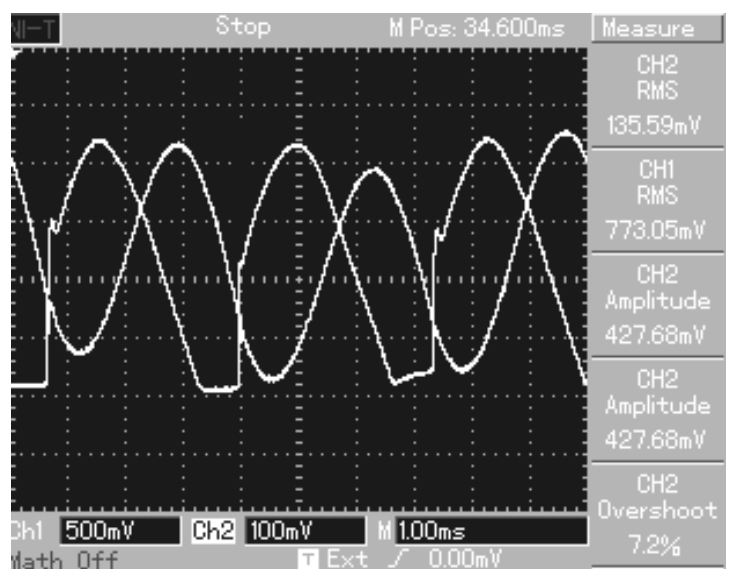

Figure 12. Ripple voltage (input and output filter).

\section{Summary}

Transformer ripple filter represent an effective alternative to conventional filters interference voltage. Preferably, it can be used for high-power DC supplies. Preferably, they are intended for stationary and industrial applications where higher matter weight compensation transformer filter. The advantage is the high long-term overload capability and effectively suppress interference voltages without large filter capacity. Additional capacitors can the level of disturbing voltage drop further.

The article was prepared with the support of specific research project SV PdF 2018/2012 - High-power DC supply with mid-frequency transformer compensation ripple voltage with high-overload current.

\section{References}

1. R. Drtina, J. Lokvenc and J. Andris. The Accessories for Your Laboratories, Part 9. - Switching supply for laboratory use. Media4u Magazine, 1, pp.55-62, (2009).

2. J. Lokvenc, R. Drtina. Power supply voltage with the transformer ripple filter. In The 11th WSEAS/ IASME International Conference on Electric Power Systems, High Voltages, Electric Machines, Penang, Malaysia, pp.60-64, (2011).

3. J. Lokvenc, R. Drtina, and M. Sobek. Three-phase high overload DC power supply voltage with the mid-frequency transformer ripple filter. In The 12th WSEAS International Conference on Electric Power Systems, High Voltages, Electric Machines, Praha. pp.29-34, (2012).

4. J. H. Harlow. Electric power transformer engineering. BOca Raton, CRC, (2007).

5. J. J. Winders. Power transformers: principles and applications. New York, Dekker, (2002). 